\title{
Phase-locked Laser Arrays Revisited
}

\author{
An Updated Review of Phase-locked Arrays \\ of Semiconductor Diode Lasers
}

$\mathrm{I}$ n January 1986 a review article on phase-locked arrays of diode lasers was published in this magazine. Now, more than a decade later, major breakthroughs have occurred, both in theory and experiment, that have allowed phase-locked arrays to meet their promise of reliable, highcontinuous-wave $(\mathrm{CW})$ power $(\cong 0.5 \mathrm{~W})$ operation in diffraction-limited beams as well as multiwatt (5-10 W) near-diffraction-limited peak-pulsed-power operation. As an update to the 1986 article, this article describes a corrected picture for the array modes, arrays of antiguides and the concept of resonant leaky-wave coupling, and relevant recent results.

\section{Overview}

By comparison with other types of highpower, coherent semiconductor-based sources ("broad area"-type master oscillator power amplifier (MOPA), unstable resonator), phase-locked arrays have some unique advantages: graceful degradation; no need for optical isolators; no need for external optics to compensate for phasefront aberrations due to thermal-and/or carrier-induced variations in the dielectric constant; and, foremost, intrinsic beam stability with drive level due to a strong, built-in, real-index profile. The consequence is that, in the long run, phase-locked arrays are bound to be fundamentally more reliable than either MOPAs or unstable resonators.

\author{
Dan Botez and Luke J. Mawst
}

At the time the original review article [1] was written, three major types of phaselocked arrays had been investigated: evanescent-wave coupled, diffraction-coupled, and Y-junction coupled (see Fig. 5 in [1]). Up to 1988 the results were not at all encouraging: maximum diffraction-limited, singlelobe powers of $\approx 50 \mathrm{~mW}$ or coherent powers (i.e., fraction of the emitted power contained within the theoretically defined diffractionlimited-beam pattern) never exceeding 100 $\mathrm{mW}$. Thus, the very purpose of fabricating arrays (to surpass the reliable power level of single-element devices) was not achieved. The real problem was that researchers had taken for granted the fact that strong nearest-neighbor coupling implies strong overall coupling. In reality, as shown in Fig. 1, nearest-neighbor coupling is "series coupling," a scheme plagued by weak overall coherence and poor intermodal discrimination [2]. Strong overall interelement cou-

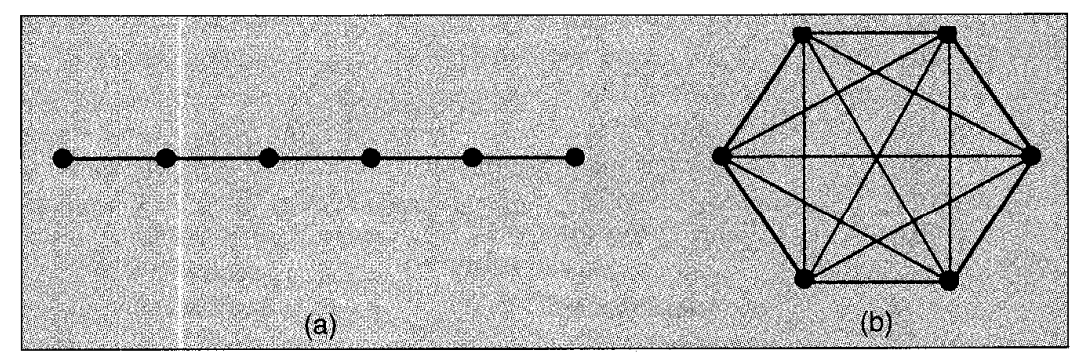

1. Types of overall interelement coupling in phase-locked arrays: (a) series coupling (nearest-neighbor coupling, coupled-mode theory); (b) parallel coupling. pling occurs only when each element equally couples to all others (so-called "parallel coupling") [2]. In turn, intermodal discrimination is maximized and full coherence becomes a system characteristic. Furthermore, parallel-coupled systems have uniform near-field intensity profiles, and are thus immune to the onset of high-ordermode oscillation at high drive levels above threshold.

Parallel coupling can be obtained in evanescent-wave-coupled devices, but only by weakening the optical-mode confinement, and thus making the devices vulnerable to thermal- and/or injected-carrier-induced variations in the dielectric constant. For both full coherence and stability it is necessary to achieve parallel coupling in structures of strong optical-mode confinement (i.e., builtin index steps $\geq 0.01$ ). As shown below, only strongly guided, leaky-wave-coupled devices can meet both conditions [3-5]. A sta- 


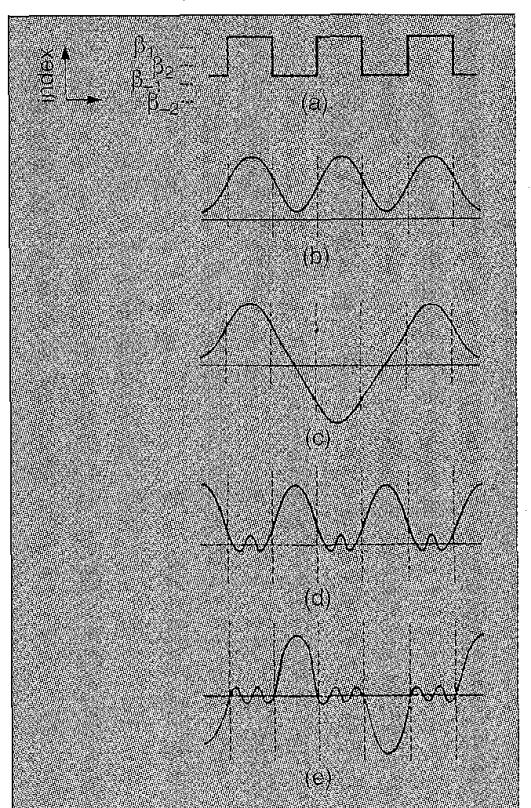

2. Modes of array of periodic real-index variations: (a) index profile; (b) in-phase evanescent-wave type; (c) out-of-phase evanescent-wave type; (d) in-phase leakywave type; (e) out-of-phase leaky-wave type. Respective propagation constants are $\beta_{1}, \beta_{2}$, $\beta-1$ and $\beta-2$.

ble, parallel-coupled source is highly desirable for systems applications since it has graceful degradation; that is, the failure or obscuration of some of its components does not affect the emitted beam pattern. Furthermore, it has been recently shown both theoretically [6] and experimentally [7] that, by contrast to series-coupled systems, parallelcoupled systems are fundamentally stable against coupling-induced instabilities.

In 1978 Scifres et al. [8] reported the first phase-locked array: a five-element gainguided device. It took eight years before Hadley [9] showed that the modes of gainguided devices are of the leaky type. Gainguided arrays have generally operated in leaky, out-of-phase (i.e., two-lobed) patterns with beamwidths many times the diffraction limit due to poor intermodal discrimination. Furthermore, gain-guided devices, being generated simply by the injected-carrier profile (Fig. 1a in [1]), are very weakly guided and thus vulnerable to thermal gradients and gain spatial-hole burning. The first real-index-guided, leakywave-coupled array (i.e., the so-called an- tiguided array) was realized in 1981 by Ackley and Engelmann [10] While the beam patterns were stable, the lobes were several times the diffraction limit, with in-phase and out-of-phase modes operating simultaneously due to lack of a mode-selection mechanism. Positive-index-guided arrays came next in array research (1983-8). Inphase, diffraction-limited-beam operation could never be obtained beyond $50 \mathrm{~mW}$ output power. Some degree of stability could be achieved in the out-of-phase operational condition such that, by 1988 , two groups $[11,12]$ reported diffraction-limited powers as high as $200 \mathrm{~mW}$.

In 1988 antiguided arrays were resurrected and, right from the first attempt, researchers obtained close to $200 \mathrm{~mW}$ diffraction-limited in-phase operation [3]. Hope for achieving high coherent powers from phase-locked arrays was rekindled, although there was no clear notion as to how to controllably obtain single-lobe operation. The breakthrough occurred in late 1988 with the discovery of resonant leaky-wave coupling [4], which allowed parallel coupling among array elements for the first time, and thus the means of achieving high-power, single-lobe, diffraction-limited operation. The experimental coherent powers quickly escalated such that to date, up to $2 \mathrm{~W}$ has been achieved in a diffraction-limited beam [13], and up to $10 \mathrm{~W}$ in a beam twice the diffraction limit [14].

\section{Array Modes Revisited}

As shown at the top of Fig. 2, a monolithic array of phase-coupled diode lasers can be described simply as a periodic variation of the real part of the refractive index. Two classes of modes characterize such a system: evanescent-type array modes (Figs. 2b and c), for which the fields are peaked in the high-index array regions; and leaky-type array modes, for which the fields are peaked in the low-index array regions (Figs. $2 \mathrm{~d}$ and e). Another distinction is that while evanescent-wave modes have effective-index values between the low and high refractive-index values, leaky modes have effective-index values below the low refractive-index value [5, 15]. For both classes of modes the locking condition is said to be "in-phase" when the fields in each element are cophasal, and "out-of-phase" when fields in adjacent elements are a $\pi$ phaseshift apart.
As described in the original paper, evanescent-type modes were the first to be analyzed, simply because it could be assumed that they arose from the superposition of individual-element wavefunctions, and thus could readily be studied via the coupledmode formalism $[16,17]$. While coupledmode theory proved quite useful in understanding early work on phase-locked arrays, it has severe limitations: it does not apply to strongly coupled systems, and it does not cover leaky-type array modes. Ironically, control of leaky modes turns out to be the key for high-power, phase-locked operation. Thus, after many years of use, coupled-mode theory has suddenly become obsolete as far as the design and analysis of high-power coherent devices is concerned.

One major reason why leaky array modes had been overlooked for so long is that they are not solutions of the popular coupled-mode theory. The other reason is that for few-element (up to five) arrays, leaky modes play a minor role since they are very lossy [15]. However, for high-power devices (10 or more elements), leaky-mode

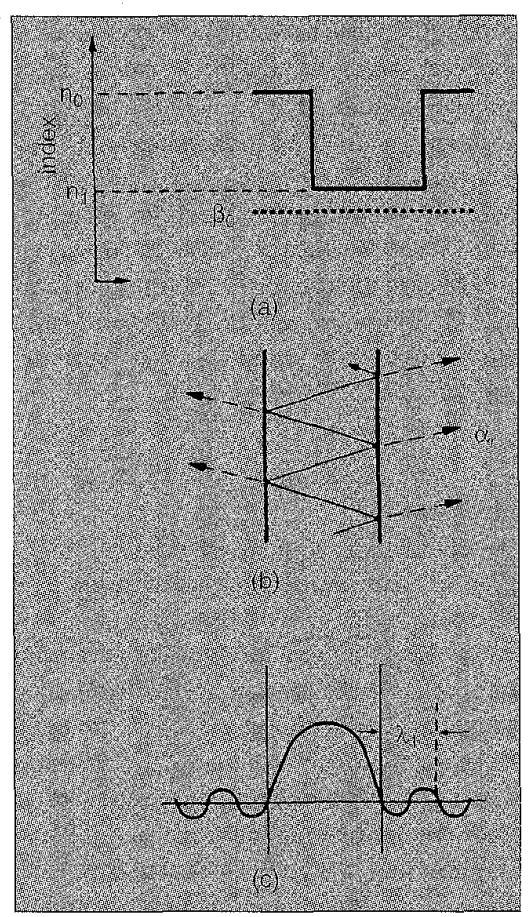

3. Schematic respresentation of real-index antiguide: (a) index profile; (b) ray-optics picture ( $\alpha_{R}$ is edge radiation loss coefficient); (c) near-field amplitude profile $\lambda_{1}$ is the leakywave periodicity in the lateral direction). 
operation is what ensures stable diffractionlimited-beam operation to high drive levels.

Evanescent-type array modes are of importance only when the modal gain in the high-index regions is higher than the modal gain in the low-index regions. Many workers [18-20] have shown that excess gain in the high-index array regions generally favors oscillation in the evanescent out-ofphase mode, in close agreement with experimental results. There is, however, one major limitation: the built-in index step, $\Delta n$ has to be below the cutoff for high-order (element) modes [19, 21]. For typical devices, $\Delta n \leq 5 \times 10^{-3}$. In turn, the devices are sensitive to gain spatial hole burning [22] and thermal gradients.

Leaky-type modes are favored to lase when gain is preferentially placed in the low-index regions $[4,23,24]$. Unlike evanescent-type modes there is no limitation on $\Delta n$; that is, no matter how high $\Delta n$ is, the modes favored to lase comprise fundamental element modes coupled in-phase or outof-phase. This fact has two important consequences. First, one can fabricate single-lobe-emitting structures of high-index steps $(0.05-0.20)$, that is, stable against thermal- and/or carrier-induced index variations. Second, it becomes clear why predictions of coupled-mode theory that excess gain in the low-index array regions [19, 21] favor the in-phase evanescent-type mode have failed. That is, the so called net-gain-between-elements approach to obtain fundamental-mode operation (see Fig. $5 b$ in [1]) was flawed. The reason is simply the fact that leaky modes could not be taken into account when using coupled-mode analysis. Ironically, the very array structures proposed by Streifer et al. for in-phase mode operation [19], when analyzed using exact theory by Fujii et al. [25], were found to primarily favor operation in an out-of-phase leaky mode. To analyze both array-mode types, for a given structure, one has to use either exact theory $[15,24,25]$ or the Blochfunction method $[23,26,27]$.

\section{Arrays of Antiguides} and Resonant Leaky-wave Coupling

The basic properties of a single real-index antiguide are shown schematically in Fig. 3 . The antiguide core has an index, no, lower than the index of the cladding, $n_{1}$. The index depression, $\Delta n$, is (1-2) $\times 10^{-3}$ for gainguided lasers and $(2-5) \times 10^{-2}$ for strongly index-guided lasers. The effective-index of

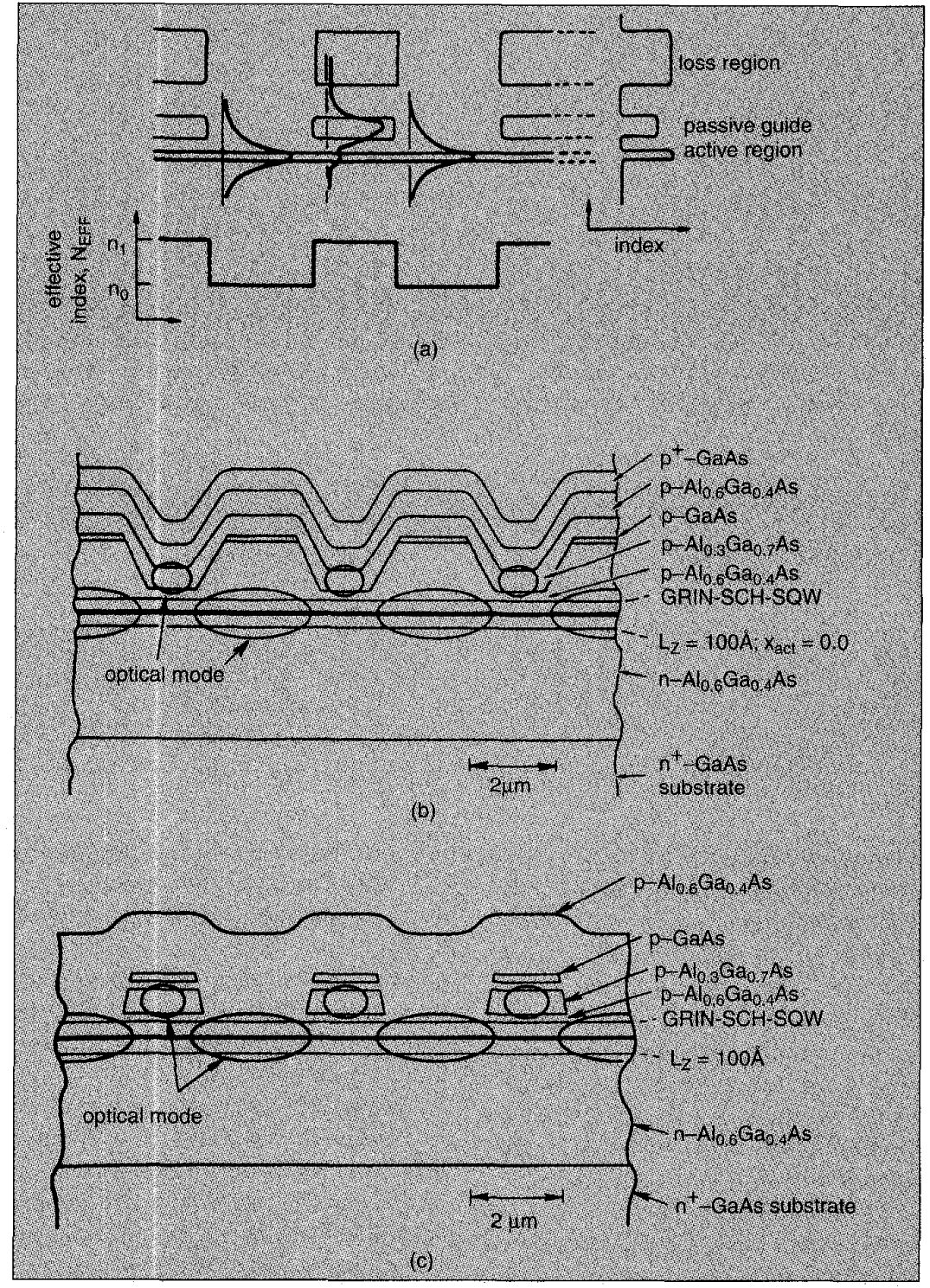

4. Schematic representation of modern types of arrays of (closely spaced) antiguides: (a) practical way of fabricating arrays of closely spaced antiguides; (b) complimentary-self-aligned (CSA) array type [15]; (c) self-aligned-stripe (SAS) array type [13, 14, 29, 30].

the fundamental mode $\beta_{0} / \mathrm{k}$ is below the index of the core. The quantum-mechanical equivalent is thus a quasibound state above a potential barrier. By contrast, the quantum-mechanical equivalent of the fundamental mode in a positive-index guide is a bound state in a potential well. Whereas in a positive-index guide, radiation is trapped via total internal reflection, in an antiguide, radiation is only partially reflected at the antiguide-core boundaries (Fig. 3b). Light refracted into the cladding layers is radiation leaking outwardly with a lateral wavelength $\lambda_{1}$ (Figure 3c) $[4,15]$ :

$$
\lambda_{1} \cong \lambda / \sqrt{2 n \Delta n+(\lambda / 2 d)^{2}},
$$

and can be thought of as a radiation loss [28]:

$$
\alpha_{R}=(l+1)^{2} \lambda^{2} / d^{3} n \sqrt{2 n \Delta n}
$$

where $d$ is the antiguide-core width, $\Delta n$ is the lateral refractive-index step, $n$ is the av- 


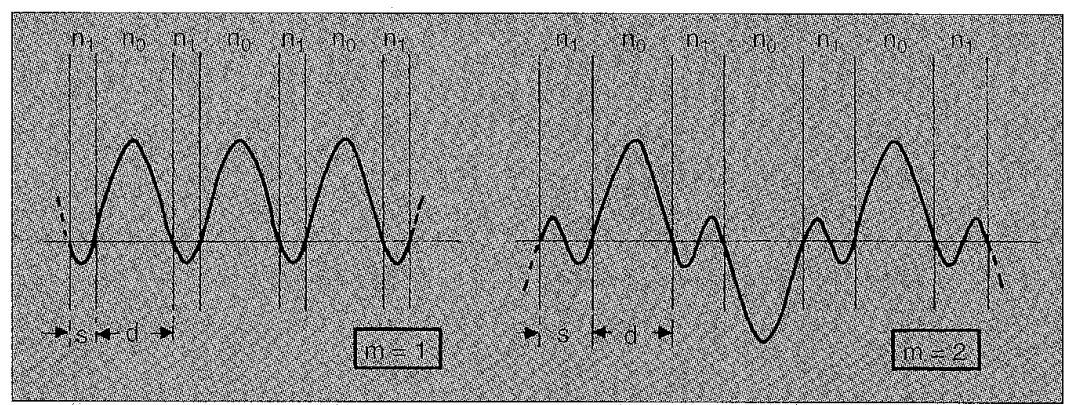

5. Near-field amplitude profiles in resonantly coupled arrays: (a) in-phase resonant mode; (b) out-of-phase resonant mode; $m$ is number of interelement near-field intensity peaks. (After [5].)

\begin{tabular}{|c|c|c|}
\hline \multirow[t]{2}{*}{ Drive condition } & \multicolumn{2}{|c|}{ Maximum power } \\
\hline & $\mathrm{DL}^{*}$ & Narrow beam \\
\hline $\mathrm{CW}$ & $0.5 \mathrm{~W}$ & $1 \mathrm{~W} ; 1.7 \mathrm{DL}$ \\
\hline Pulsed & $2.1 \mathrm{~W}$ & $10 \mathrm{~W} ; 2.0 \mathrm{DL}$ \\
\hline
\end{tabular}

erage index value, $\lambda$ is the vacuum wavelength, and $l$ is the (lateral) mode number. For typical structures $(d=3 \mu \mathrm{m}, \Delta n=2-3 \mathrm{x}$ $10^{-2}$ ) at $\lambda=0.85 \mu \mathrm{m}$, typical $\lambda_{1}$ and $\alpha_{R}$ values are $2 \mu \mathrm{m}$ and $100 \mathrm{~cm}^{-1}$, respectively. Since $\alpha_{R} \propto(l+1)^{2}$, the antiguide acts as a lateral-mode discriminator. For a proper mode to exist, $\alpha_{R}$ has to be compensated for by gain in the antiguide core [28]. Single antiguides have already been used for quite some time in $\mathrm{CO}_{2}$ "waveguide" lasers.

Historically, the first arrays of antiguided lasers were gain-guided arrays [8] (Figure 1a in [1]), since an array of currentinjecting stripe contacts provides an array of (carrier-induced) index depressions for which the gain is highest in the depressedindex regions. While for a single antiguide the radiation losses can be quite high [28], closely spacing antiguides in linear arrays significantly reduces the device losses [3-5] since radiation leakage from individual elements mainly serves the purpose of coupling the array elements.

The first real-index antiguided array was realized by Ackley and Engelmann [10]: an array of buried heterostructure $(\mathrm{BH})$ lasers designed such that the interelement regions had higher refractive indices than the effective refractive indices in the buried active mesas. Since the high-index interelement regions had no gain, only leaky array modes could lase. The device showed definite evidence of phase locking (in-phase and out-ofphase) but had relatively high threshold-current densities $\left(5-7 \mathrm{kA} / \mathrm{cm}^{2}\right)$ since the elements were spaced far apart (13-15 $\mu \mathrm{m}$ ), thus not allowing for effective leaky-wave coupling.

For practical devices the high-index regions have to be relatively narrow $(1-3 \mu \mathrm{m})$, which is virtually impossible to achieve using BH-type fabrication techniques. Instead, one can fabricate narrow, high-effective-index regions by periodically placing high-index waveguides in close proximity $(0.1-0.2$ $\mu \mathrm{m}$ ) to the active region $[3-5,13]$ (see Fig. 4). In the newly created regions the fundamental transverse mode is primarily confined to the passive guide layer; that is, between the antiguided-array elements the modal gain is low. To further suppress oscillation of evanescent-wave modes an optically absorbing material can be placed between elements (Fig. 4a).

The first closely spaced, real-index antiguided array was realized by liquid-phase epitaxy (LPE) over a patterned substrate [3]. Devices made in recent years are fabricated by metal-organic chemical vapor deposition (MOCVD) and can be classified into two types: the complimentary-self-aligned
(CSA) stripe array [15] (Fig. 4b); and the self-aligned-stripe (SAS) array [13, 14, 29, 30] (Fig. 4c). In CSA-type arrays preferential chemical etching and MOCVD regrowth occur in the interelement regions. For SAStype arrays the interelement regions are built-in during the initial growth, and then etching and MOCVD regrowth occur in the element regions. A most recent approach, which does not involve regrowth, is the formation of low-index, high-gain regions via preferential $Z n$-diffusion disordering of a superlattice upper cladding layer [49].

Owing to lateral radiation, a single antiguide can be thought of as a generator of laterally propagating traveling. waves of wavelength $\lambda_{1}$ (Fig. 3). Then, in an array of antiguides, elements will resonantly couple in-phase or out-of-phase when the interelement spacings correspond to an odd or even integral number of (lateral) half-wavelengths $\left(\lambda_{1} / 2\right)$, respectively (see Fig. 5). The resonance condition is

$$
\begin{aligned}
& s=\mathrm{m} \lambda_{1} / 2 \\
& \mathrm{~m}=\text { odd } \quad \text { resonant in - phase mode } \\
& \mathrm{m}=\text { even }
\end{aligned}
$$

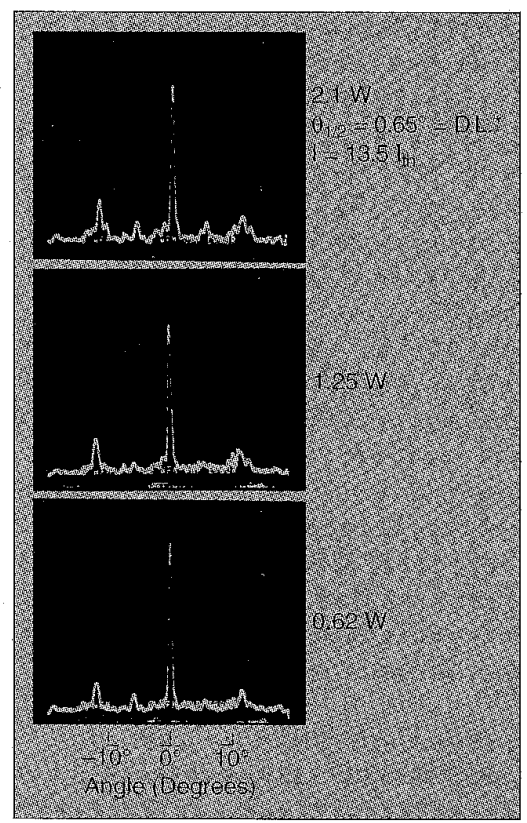

6. Lateral far-field beam patterns at several power levels for a resonant array with a monolithic Talbot filter. A diffraction-limited beam is maintained above the $2 W$ output power level. $\left(\right.$ After [13].) ${ }^{*} D . L .=$ diffraction limited 


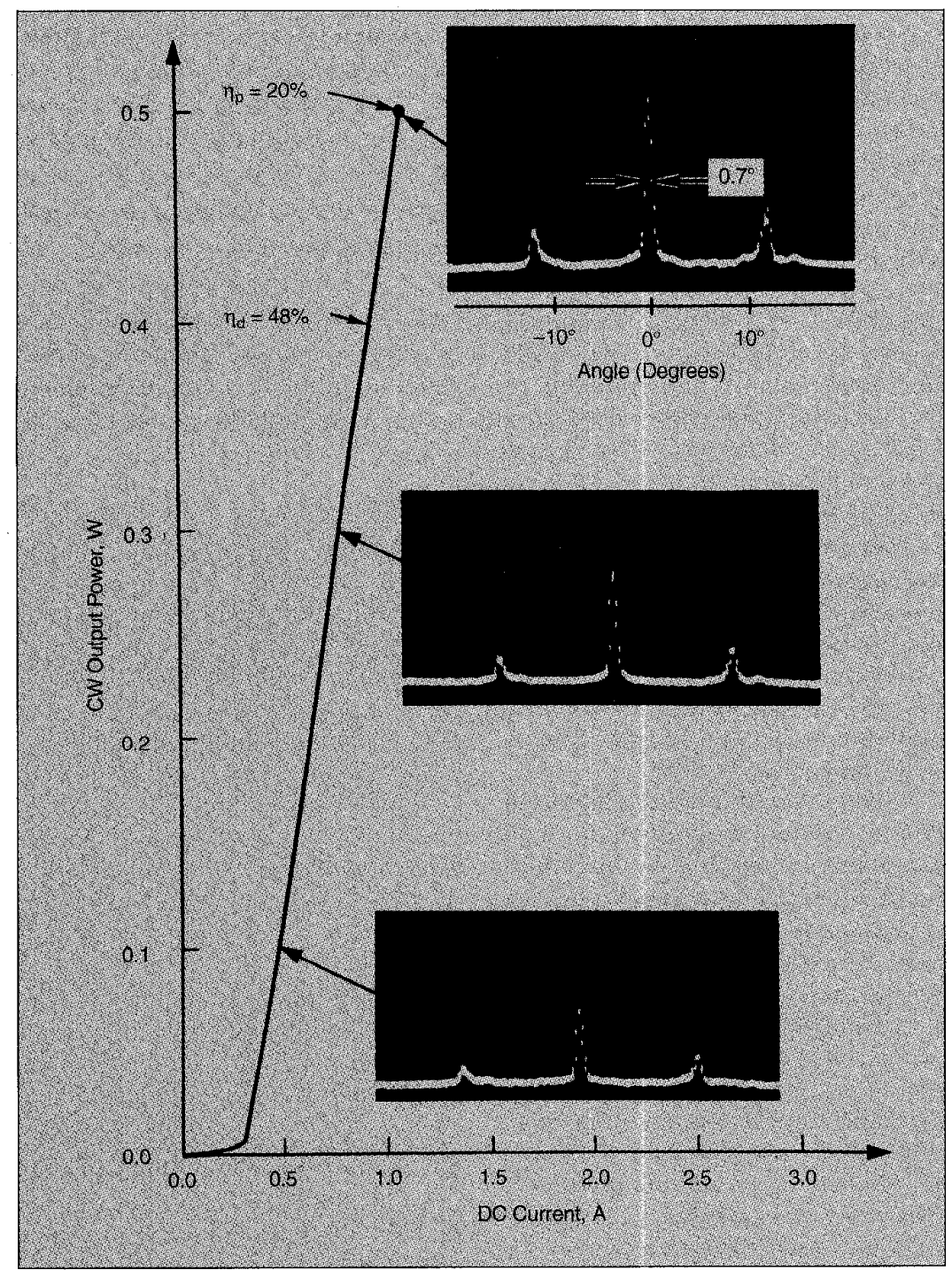

7. CW operation of optimized 20-element ROW array: light-current characteristic and lateral far-field patterns at various power levels. Beam pattern is diffraction-limited up to $0.5 \mathrm{~W}$.

where $s$ is the interelement spacing. Typical $s$ values are $1 \mu \mathrm{m}$. Then, for in-phase-mode resonance, $\lambda_{1}=2 \mu \mathrm{m}$. The lateral resonance effect in antiguided arrays is quite similar to the 2nd-order Bragg resonance in DFB-type structures [31]. In fact a resonant antiguided array is a lateral complex-coupled 2nd-order DFB structure.

When the resonance condition is met, the interelement spacings become Fabry-Perot resonators in the resonance condition [5]. Then each element can equally couple to all others, thus realizing, for the first time, the dream of researchers in the phase-locked-array field: parallel coupling (Fig. 1b). Reso- tion can be achieved by employing intracavity Talbot-type spatial filters [34, 35]. It was in fact a 20-element array with Talbottype spatial filters [36] that allowed, in 1990, the "breaking" of the $1 \mathrm{~W}$ coherent-power barrier for monolithic semiconductor diode lasers.

\section{Relevant Recent Results}

In pulsed operation, 20-element devices have demonstrated diffraction-limited beams to $1.5 \mathrm{~W}$ and 10.7 times the threshold [37], and to $2.1 \mathrm{~W}$ and 13.5 times the threshold [13] (Fig. 6). At the $2.1 \mathrm{~W}$ power level only $1.6 \mathrm{~W}$ is coherent uniphase power and the main lobe contains $1.15 \mathrm{~W}$ (so-called "power in the bucket"). Actually, close to $100 \%$ of the coherent power can be garnered in the main lobe by using aperture-filling optical techniques [38]. The fact that diffraction-limited beams can be maintained to very high drive levels is a direct consequence of the inherent self-stabilization of the in-phase resonant mode with increasing drive level [39].

When carrier diffusion is taken into account [40], it has been shown that for devices with $\Delta n \cong 0.025$, the adjacent mode reaches threshold (i.e., multimode operation starts) at more than seven times the threshold of the in-phase mode. Furthermore, if the index step is increased to $\approx 0.16$, the effect of gain spatial hole burning is negligible, such that one can achieve single-(in-phase)mode operation to drive levels $>15$ times the threshold, powers of $\approx 3 \mathrm{~W}$, in beams with $\cong 70 \%$ of the energy in the main lobe [40].

In CW operation, one is limited by thermal effects. The best result in a purely diffraction-limited beam is $0.5 \mathrm{~W} \mathrm{CW}$ from a 20 -element device of $80 \mu \mathrm{m}$-wide aperture (Fig. 7). For $1000 \mu \mathrm{m}$-long devices with HR and AR facet coatings ( 98 and $4 \%$ reflectivity), slope efficiencies of $48-50 \%$ are observed and the power conversion efficiency reaches values in the $20-25 \%$ range at $0.5 \mathrm{~W}$ output. Preliminary lifetests at $0.5 \mathrm{~W} \mathrm{CW}$ output show room-temperature extrapolated lifetimes in excess of $5000 \mathrm{~h}$ [41]. Thus the prediction of the original article [1] (see "Conclusion" section) has been fulfilled.

Recently, by using large-aperture (120 $\mu \mathrm{m}), 20$-element structures of 5 to 1 element/interelement width ratio, we obtained [42] $1 \mathrm{~W}$ CW operation in a beam 1.7 times the diffraction limit (Fig. 8). Close to threshold $(0.1 \mathrm{~W})$ the beam is diffraction limited, and $75 \%$ of the energy resides in the main 


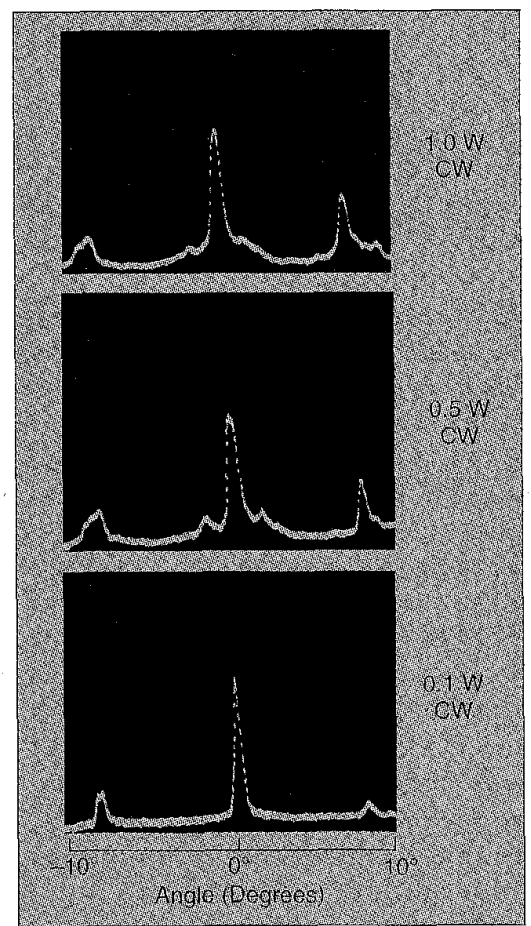

8. CW far-field patterns of 20-element ROW array with $5 \mathrm{\mu m}$-wide elements and $1 \mathrm{\mu m}$ wide interelements at various power levels. (After [42].)

lobe. The $1 \mathrm{~W}$ value represents the highest $\mathrm{CW}$ coherent power achieved to date from any type of fully monolithic diode emitters (i.e., without phase-correcting optics).

The results presented so far are for $\mathrm{Al}$ GaAs/GaAs structures $(\lambda=0.84-0.86 \mu \mathrm{m})$. Antiguided arrays have also been made from strained-layer quantum-well material $(\lambda=$

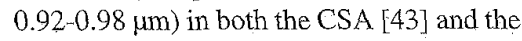
SAS-type array configurations: InGaAs/In$\mathrm{GaP} / \mathrm{GaAs}$ [14] and $\mathrm{InGaAs} / \mathrm{AlGaAs} / \mathrm{GaAs}$ $[29,30]$. Resonant devices were achieved only with the CSA configuration, and provided $1 \mathrm{~W}$ pulsed diffraction-limited-beam operation [43]. From SAS-type devices Major et al. [30] have demonstrated stable and efficient $\mathrm{CW}$ operation to $0.5 \mathrm{~W}$ in a beam 1.5 times the diffraction limit.

Nonresonant devices typically have beams 2 to 3 times the diffraction limit. First of all, the in-phase mode has a raised-cosineshaped envelope and thus gain spatial hole burning is nonuniform across the device causing mode self-focusing $[39,40]$, similar to evanescent-wave coupled devices [22]. Then adjacent modes can readily reach threshold and the beamwidth increases to 2 to 3 times the diffraction limit. The combi nation of the in-phase mode and one or two adjacent modes uses the avaitable gain efficiently, thus not allowing other modes to come in. This explains why stable beams can be maintained to very high drive levels and peak powers: $5 \mathrm{~W}$ to 45 times the threshold from $80 \mu \mathrm{m}$-wide aperture devices [36], 10 $\mathrm{W}$ from $200 \mu \mathrm{m}$-wide aperture devices [14] (Fig. 9), and $11.5 \mathrm{~W}$ from $185 \mu \mathrm{m}$-wide aperture devices [44]. Particularly notable is the achievement of a beam pattern 2 times the diffraction limit with $6 \mathrm{~W}$ in the central lobe [14] (Fig. 9) from an Al-free structure $(\lambda=0.98 \mu \mathrm{m})$.

Bloch-function array analysis has been recently extended $[45,46]$ from infinite-extent arrays to finite-extent arrays. Analytical formulae can then be obtained for the resonant-array-mode loss [45] intermodal discrimination [46], and near- and far-field patterns [47]. Thus, we have now a complete analytical model that should allow for straightforward device design.

Finally it has been found both theoretically $[6,7]$ and experimentally [7] that ROW arrays do not suffer from the coupling-induced instabilities that generally plague series-coupled devices such as evanescent-wave coupled and Y-junction coupled arrays. Quiescent behavior up to $0.45 \mathrm{~W}$ cw power and 3.4 times the threshold, in near diffraction-limited beams, has been recorded from ROW arrays with negligible interelement loss [7]. (Too high a value for interelement loss causes saturableabsorption-induced self-pulsations) [7]. This is the first time that a phase-locked array has been found to be temporally stable to substantial powers and drive levels above threshold. Li and Erneux [6] have shown that the intrinsic stability of ROW arrays is due to parallel coupling.

\section{Conclusions - A Decade Later}

Arrays based on coupling of positive-index guides, although not successful for highpower coherent applications, may still be of use. One application could be controlled beam steering. For instance, one could create a phase ramp across the aperture of an evanescent-wave-coupled array by simply tailoring the injected-carrier profile. By varying currents through separate contact pads, the beam pattern could then be made to shift controllably.

It is now apparent that positive-indexguided devices were just one stage in array development. What has finally made phaselocked arrays a success has been the discovery of a mechanism for selecting in-phase leaky modes of antiguided structures. Paral lel coupling in strong index-guided structures has allowed ROW arrays to reach stable diffraction-limited operation to powers 20-30 times higher than for other array types. This finally fulfills the phase-locked arrays promise of vastly improved coherent power by comparison with single-element devices. Table 1 summarizes the best results to date.

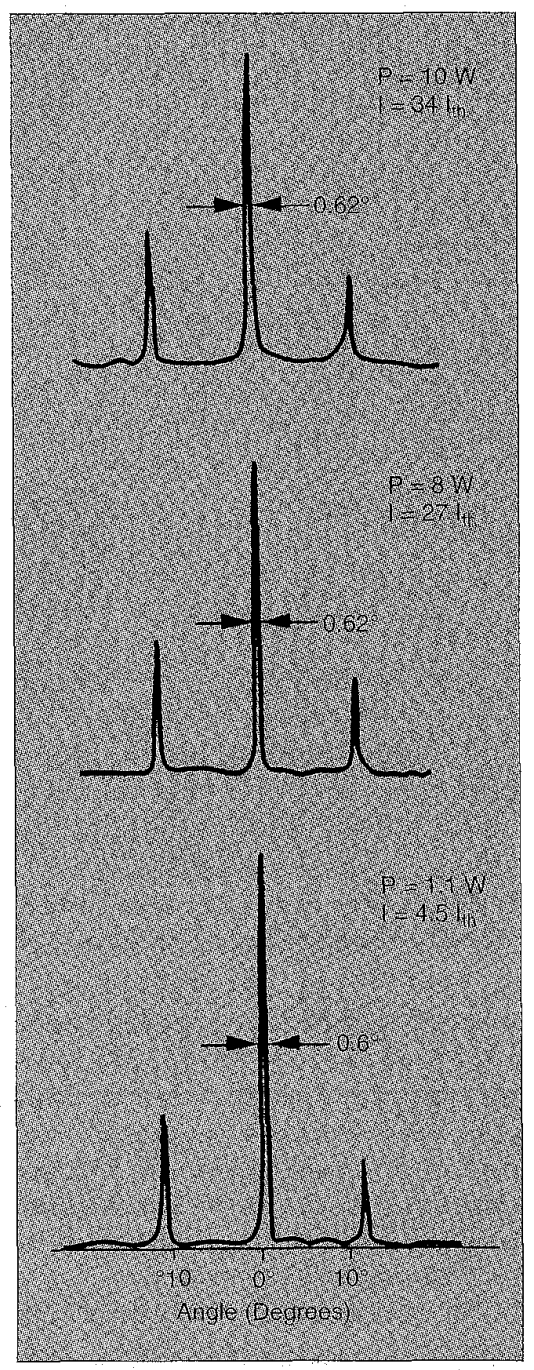

9. Pulsed far-field patterns of 40 element antiguided array of Al-free $(\lambda=0.98 \mu \mathrm{m})$ diode lasers. The beam is 2.0 times the diffraction limit up to 34 times the threshold (10 W). (After [14].) 
In the future, for edge-emitting coherent arrays, the eventual limitation may just be thermal; that is , just as for incoherent devices, maximum emitting apertures would be $400-500 \mu \mathrm{m}$ wide. Thus, the projected maximum diffraction-limited $\mathrm{CW}$ powers are in the $3-5 \mathrm{~W}$ range. Beyond $5 \mathrm{~W}$, one will have to resort to 2-D surface-emitting arrays. A 2-D surface-emitting ROW array has already been demonstrated [48]. Interunit coupling occurs via resonant leaky-wave coupling, while radiation outcoupling is realized via $45^{\circ}$ micromachined turning mirrors. Preliminary results from nine-unit arrays are quite encouraging: $3.9 \mathrm{~W}$ with $45 \%$ visibility and $6 \mathrm{~W}$ with $33 \%$ visibility [48]. For a very large number of units $(\geq 100)$ there is always the concern that the device may operate in several mutually coherent regions and thus, to control hundreds of emitters, a master oscillator (i.e., injection locking) may be required. All in all we foresee that 2-D ROW arrays will eventually be capable of $\mathrm{CW}$ coherent powers of the order of $50 \mathrm{~W}$.

\section{Further Reading}

In-depth reviews of work on monolithic phase-locked diode-laser arrays can be found in the article: D. Botez, "High-power Monolithic Phase-locked Arrays of Antiguided Semiconductor Diode Lasers," Proc. Inst. Elec. Eng. Part J, Optoelectronics, vol. 139, pp. 14-23, Feb. 1992.; and in Chapter I of the book Diode Laser Arrays eds. D. Botez and D.R. Scifres, Cambridge University Press, Cambridge, July 1994.

Dan Botez is the Philip Dunham Reed Professor of Electrical Engineering and Director of the Reed Center for Photonics at the University of Wisconsin-Madison. Luke J. Mawst is an Assistant Professor at the University of Wisconsin-Madison, where he is involved in the development of semiconductor laser structures using the InGaP-based material system.

C\&D

\section{References}

1. D. Botez and D.E. Ackley, "Phase-locked Arrays of Semiconductor Diode Lasers, "IEEE Circuits and Devices Magazine, vol. 2, pp. 8-17, Jan. 1986.

2. W.J. Fader and G.E. Palma, "Normal modes of $N$ coupled lasers," Opt. Lett., vol. 10, pp. 381-383, 1985.

3. D. Botez, L. Mawst, P. Hayashida, G. Peterson, and T.J. Roth, "High-power diffraction-limited- beam operation from phase-locked diode-laser arrays of closely spaced 'leaky' waveguides (antiguides)," Appl. Phys. Lett., vol. 53, pp. 464-466, Aug. 1988.

4. D. Botez, L.J. Mawst, and G. Peterson, "Resonant leaky-wave coupling in linear arrays of antiguides," Electron. Lett., vol. 24, pp. 1328-1330, Oct. 1988.

5. D. Botez, L.J. Mawst, G. Peterson, and T.J. Roth, "Resonant optical transmission and coupling in phase-locked diode-laser arrays of antiguides: The resonant-optical-waveguide array," Appl. Phys. Lett., vol. 54, pp. 2183-2185, May 1989

6. Ruo-ding Li and Thomas Erneux, "Stability conditions for coupled lasers: series coupling versus parallel coupling," Opt. Comm., vol. 99, pp. 196-200, 1 June 1993

7. S. Ramanujan, H.G. Winful, M. Felisky, R.K. DeFreez, D. Botez, M. Jansen and P. Wisseman, "Temporal Behavior of Resonant-OpticalWaveguide Phase-Locked Diode Laser Arrays," Appl. Phys. Lett., vol. 64, pp. 827-829, Feb. 1994. 8. D.R. Scifres, R.D. Burnham, and W. Streifer, "Phase Locked Semiconductor Laser Array," Appl. Phys. Lett., vol. 33, pp. 1015-1017, Dec. 1978.

9. G.R. Hadley, J.P. Hohimer and A. Owyoung, "High-order $(v>10)$ eigenmodes in ten-stripe gain-guided diode laser arrays," Appl. Phys. Lett., vol. 49, pp. 684-686, 1986.

10. D.E. Ackley and R.W.H. Engelmann, "High Power Leaky Mode Multiple Stripe Laser," Appl. Phys. Lett., vol. 39, pp. 27-29, July 1981.

11. D. Botez, P. Hayashida, L.J. Mawst and T.J. Roth, "Diffraction-limited-beam, high-power operation from X-junction coupled phase-locked arrays of AlGaAs/GaAs diode lasers," Appl. Phys. Lett., vol. 53, pp. 1366-1368, Oct. 1988.

12. H. Hosoba, M. Matsumoto, S. Matsui, S. Yano and T. Hijikata, "Phased array laser diode -SAWTOOTH channelled array," The Review of Laser Engineering, vol. 17, pp. 32-42, Jan. 1989 (in Japanese).

13. L.J. Mawst, D. Botez, C. Zmudzinski, M. Jansen, C. Tu, T.J. Roth, and J. Yun, "Resonant self-aligned-stripe antiguided diode laser array," Appl. Phys. Lett., vol. 60, pp. 668-670, 1992.

14. H.Yang, L.J. Mawst, M. Nesnidal, J. Lopez, A. Bhattacharya, and D. Botez, "10 W Near-Diffraction-Limited Pulsed Power from Al-free Phase-Locked Antiguided Arrays," Tech. Dig. LEOS '96 Annual Meeting, Paper MM3, Nov. 18-20th 1996, Boston, MA.

15. D. Botez, L.J. Mawst, G.Peterson, and T.J. Roth, "Phase-locked arrays of antiguides: Modal content and discrimination," IEEE J. Quantum Electron., vol. QE-26, pp. 482-495, March 1990. 16. J.K. Butler, D.E. Ackley, and D. Botez, "Coupled Mode Analysis of Phase-Locked Injection Laser Arrays," Appl. Phys. Lett., vol. 44, pp.
293-295, Feb. 1984, see also Appl. Phys. Lett., vol. 44, p. 935, May 1984.

17. E. Kapon, J. Katz, and A. Yariv, "Supermode Analysis of Phase-Locked Arrays of Semiconductor Lasers," Opt. Lett., vol. 10, pp. 125-127, Apr. 1984.

18. S.R. Chinn and R.J. Spiers, "Modal Gain in Coupled-Stripe Lasers," IEEE J. Quantum Electron., vol. QE-20, no. 4, pp. 358-363, Apr. 1984. 19. W. Streifer, A. Hardy, R.D. Burnham, and D.R. Scifres, "Single-Lobe Phased-Array Diode Lasers," Electron. Lett., vol. 21, no. 3, pp. 118120, Jan. 1985.

20. J.K. Butler, D.E. Ackley and M. Ettenberg, "Coupled mode analysis of gain and wavelength oscillation characteristics of diode laser phased arrays," IEEE J. Quantum Electron., vol. QE-21, pp. 458-464, May 1985.

21. W. Streifer, A. Hardy, R.D. Burnham, R.L. Thornton, and D.R. Scifres, "Criteria for Design of Single-Lobe Phased-Array Diode Lasers," Electron. Lett., vol. 21, no. 11, pp. 505-506, May 1985.

22. Kuo-Liang Chen and Shyh Wang, "Spatial hole burning problems in evanescently coupled semiconductor laser arrays," Appl. Phys. Lett., vol. 47, pp. 555-557, Sept. 1985.

23. P.G. Eliseev, R.F. Nabiev and Yu. M. Popov, "Analysis of laser-structure anisotropic semiconductors by the Bloch-function method," J. Sov. Las. Res., vol. 10, 6, pp. 449-460, 1989.

24. G.R. Hadley, "Two-dimensional waveguide modeling of leaky-mode arrays," Opt. Lett., vol. 14, pp.859-861, Oct. 1989.

25. H. Fujii, I. Suemune and M. Yamanishi, "Analysis of transverse modes of phase-locked multi-stripe lasers," Electron. Lett., vol. 21, pp. 713-714, Aug. 1985.

26. D. Botez and T. Holcomb, "Bloch-function analysis of resonant arrays of antiguided diode lasers," Appl. Phys. Lett., vol. 60, pp. 539-541, Feb. 1992.

27. R.F. Nabiev and A.I. Onishchenko, "Laterally coupled periodic semiconductor laser structures: Bloch function analysis," IEEE J. Quantum Electron., vol. 28, pp. 2024-2032, Oct. 1992.

28. R.W. Engelmann and D. Kerps, "Leaky modes in active three-layer slab waveguides," Proc. Inst. Elec. Eng. Part I, vol. 127, pp.330-336, Dec. 1980.

29. T.H. Shiau, S. Sun, C.F. Schaus, K. Zheng and G.R. Hadley, "Highly stable strained layer leakymode diode laser arrays," IEEE Photonics Technol. Lett., vol. 2, pp. 534-536, 1990.

30. J.S. Major, Jr., D. Mehuys, D.F. Welch, and D. Scifres, "High power high efficiency antiguide laser arrays," Appl. Phys. Lett., vol. 59, pp. 22102212 , Oct. 1991

31. C.A. Zmudzinski, D. Botez, and L.J. Mawst, "Simple description of laterally resonant, distributed-feedback-like modes of arrays of an- 
tiguides," Appl. Phys. Lett., vol. 60, pp. 10491051, March 1992.

32. D. Botez and L.J. Mawst, " $\Gamma$ effect: Key intermodal-discrimination mechanism in arrays of antiguided diode lasers," Appl. Phys. Lett., vol. 60, pp. 3096-3098, 1992.

33. G.R. Hadley, "Index-guided arrays with a large index step," Opt. Lett., vol. 14, pp. 308-310, 1989.

34. L.J. Mawst, D. Botez, T.J. Roth, W.W. Simmons, G. Peterson, M. Jansen, J.Z. Wilcox, and J.J. Yang, "Phase-locked array of antiguided lasers with monolithic spatial filter," Electron. Lett., vol. 25, pp. 365-366, 1989.

35. P.D. Van Eijk, M. Reglat, G. Vasilieff, G.J.M. Krijnen, A. Driessen, and A.J, Mouthaan, "Analysis of modal behavior of an antiguide diode laser array with Talbot filter," IEEE J. Lightwave Technol., vol. LT-9, pp. 629-634, 1991.

36. D. Botez, M. Jansen, L.J. Mawst, G. Peterson and T.J. Roth, "Watt-range, coherent, uniphase powers from phase-locked arrays of antiguided diode lasers," Appl. Phys. Lett., vol. 58, pp. 20702072, 1991 .

37. L.J. Mawst, D. Botez, M. Jansen, T.J. Roth and J. Rozenbergs, "1.5 W diffraction-limitedbeam operation from resonant-optical-waveguide (ROW) array," Electron. Lett., vol. 27, pp. 369371, 1991.
38. J.R. Leger, G.J. Swanson and M. Holtz, "Efficient side lobe suppression of laser diode arrays," Appl. Phys. Lett., vol. 50, pp. 1044-1046, 1987.

39. R.F. Nabiev, P. Yeh and D. Botez, "Self-stabilization of the fundamental in-phase mode in resonant antiguided laser arrays," Appl. Phys. Lett., vol: 62, pp. 916-918, March 1993.

40. R.F. Nabiev and D. Botez, "Comprehensive Above-Threshold Analysis of Antiguided Diode Laser Arrays," IEEE J. Select. Topics Quantum Electron, vol. 1, No. 2, pp. 138-149, June 1995.

41. L.J. Mawst, D. Botez, M. Jansen, T.J. Roth, C. Zmudzinski, C. Tu and J. Yun, "Resonant-optical-waveguide antiguided diode laser arrays," SPIE Proceedings OE-LASE "92 Meeting, vol. 1634, pp. 2-12, 1992

42. C. Zmudzinski, D. Botez, and L.J. Mawst, "Coherent, One-Watt Operation of Large-Aperture Resonant Arrays of Antiguided Diode Lasers," Appl. Phys. Leitt, vol. 62, pp. 2914-2916, 1993.

43. C. Zmudzinski, L.J. Mawst, D. Botez, C. Tu and C.A. Wang, "I W Diffraction-limited-beam operation of resonant-optical-waveguide diode laser arrays at $0.98 \mu \mathrm{m}$," Electron. Lett., vol. 28 , pp. 1543-1545, July 1992.

44. J.S. Major, Jr., D. Mehuys and D.F. Welch, "11.5 W, Nearly Diffraction-Limited Pulsed Op- eration of an Antiguided Laser Diode Array," Electron. Lett., vol. 28, pp. 1101-1103, June 1992. 45. D. Botez and A.P. Napartovich, "Phaselocked Arrays of Antiguides: Analytical Theory," IEEE J. Quantum Electron., vol. 30, pp. 975-980, April 1994; Erratum, vol. 32, Dec. 1996.

46. D. Botez, A. Napartovich and C. Zmudzinski, "Phase-Locked Array of Antiguides: Analytical Theory - II," IEEE J. Quantum Electron., vol. 31, pp. 244-253, Feb. 1995.

47. A. Napartovich, D. Botez, "Analytical Theory of the Array Modes of Resonant Arrays of Antiguides," Kvantovaya Electronika (Russian Journal of Quantum Electronics), vol. 26, No. 8, pp. 670, August 1996.

48. L.J. Mawst, D. Botez, M. Jansen, C. Zmudzinski, S.S. Ou, M. Sergant, T.J. Roth, C. Tu, G. Peterson and J.J. Yang, "Two-Dimensional Surface-Emitting Leaky-Wave Coupled Laser Arrays," IEEE J. Quantum Electron., vol. 29, pp. 1906-1918, June 1993.

49. J.M. Gray and J.M. Marsh, "850-nm antiguided laser array fabricated using a zinc disordered superlattice," Tech. Dig. Conference on Lasers and Electro-Optics '96, vol. 9, p. 78, Anaheim, CA, June 2-7 1996

\section{Gigascale Integration \\ (continued from page 24)}

The prospects of scaling future species of MOSFETs to $25 \mathrm{~nm}$ minimum feature sizes (and indeed beyond) are promising. Furthermore, between the $25 \mathrm{~nm}$ MOSFET and the $0.118 \mathrm{~nm}$ tetrahedral radius of an $\mathrm{Si}$ atom lie still another two decades of opportunity to scale dimensions; or about as much as we have "consumed" so far. Discounting any sub- $25 \mathrm{~nm}$ breakthroughs, between the $125 \mathrm{~nm}$ and the $25 \mathrm{~nm}$ generations of chips we can forecast four or five intermediate generations, which should carry us to the trillion transistor chip or terascale integration (TSI). Therefore, following our anticipated achievement of the $125 \mathrm{~nm}$ generation in about a decade, at a rate of three to six years per succeeding generation, we should expect scaling to continue into the 2020 2030 timeframe.

\section{Acknowledgments}

This work was supported by the Defense Advanced Research Projects Agency (Contract: F336159C1623) and the Semiconduc- tor Research Corporation (Contract: SJ374).

James Meindl is a the J.M. Pettit Chair Professor of Microelectronics at the Georgia Institute of Technology School of Electrical and Computer Engineering in Atlanta, Georgia.

C\&D

\section{References}

1. The National Technology Roadmap for Semiconductors, Semiconductor Industry Association, 1994.

2. R.W. Keyes, "Physical Limits in Digital Electronics," Proc. IEEE, Vol. 63, No. 5, pp. 740-767, May 1975.

3. R.W. Keyes, "The Evolution of Digital Electronics Towards VLSI," IEEE JSSC, Vol. SC-14 No. 2, pp. 193-201, April 1979.

4. J:D. Meind1, "Theoretical, Practical and Analogical Limits in ULSI," IEEE IEDM Tech. Dig., pp. 8-13, 1983.

5. J.D. Meindl, "Low Power Microelectronics: Retrospect and Prospect," Proc. IEEE, Vol. 83, No. 4, pp. 619-635, April 1995.
6. A.J. Bhavnagarwala, V.K. De, B. Austin, J.D. Meindl, "Circuit Techniques for Low Power CMOS GSI," 1996 International Symposium on Low Power Electronics and Design: Digest of Technical Papers, pp. 193-196, August 1996.

7. G.A. Sai-Halasz, "Performance Trends in High-End Processors," Proc. IEEE, Vol. 83, No. 1, pp. 20-36. Jan. 1995.

8. H.T. Kung, "Why Systolic Architectures,"IEEE Computer, pp. 37-46, Jan. 1982.

9. J. Davis, V.K. De, J.D. Meindl, "Optimal Low Power Interconnect Networks," 1996 Symposium on VLSI Technology: Digest of Technical Papers, pp. 78-79, June 1996.

10. T. Ohmi, "Scientific Semiconductor Manufacturing Based on Ultraclean Processing Concept," Inter. Conf. on Advanced Microelectronic Devices and Processing, pp. 3-22, Sendai, Japan, Mar. 1994

11. R.W. Keyes, "The Effect of Randomness in the Distribution of Impurity Atoms on FET Thresholds," App. Phys., 8, pp. 251-259, 1975.

12. V.K. De, et al., "Random MOSFET Parameter Fluctuation Limits to Gigascale Integration," Dig. of VLSI Tech. Sym., pp. 198-199, 1996. 